Article

\title{
Synthesis and Characterization of Chitosan/Reduced Graphene Oxide Hybrid Composites
}

\author{
Karolina Kosowska, Patrycja Domalik-Pyzik* (D, Małgorzata Krok-Borkowicz and Jan Chłopek \\ Department of Biomaterials and Composites, Faculty of Materials Science and Ceramics, AGH University of \\ Science and Technology, Al. Mickiewicza 30, 30-059 Krakow, Poland \\ * Correspondence: pdomalik@agh.edu.pl; Tel.: +48-12-617-37-59
}

Received: 16 May 2019; Accepted: 26 June 2019; Published: 28 June 2019

\begin{abstract}
Graphene family materials (GFM) are currently considered to be one of the most interesting nanomaterials with a wide range of application. They can also be used as modifiers of polymer matrices to develop composite materials with favorable properties. In this study, hybrid nanocomposites based on chitosan (CS) and reduced graphene oxide $(\mathrm{rGO})$ were fabricated for potential use in bone tissue engineering. CS/rGO hydrogels were prepared by simultaneous reduction and composite formation in acetic acid or lactic acid and crosslinked with a natural agent-tannic acid (TAc). A broad spectrum of research methods was applied in order to thoroughly characterize both the components and the composite systems, i.e., X-ray Photoelectron Spectroscopy, X-ray Diffractometry, Attenuated Total Reflection Fourier-Transform Infrared Spectroscopy, Scanning Electron Microscopy, ninhydrin assay, mechanical testing, in vitro degradation and bioactivity study, wettability, and, finally, cytocompatibility. The composites formed through the self-assembly of CS chains and exfoliated rGO sheets. Obtained results allowed also to conclude that the type of solvent used impacts the polymer structure and its ability to interact with rGO sheets and the mechanical properties of the composites. Both rGO and TAc acted as crosslinkers of the polymer chains. This study shows that the developed materials demonstrate the potential for use in bone tissue engineering. The next step should be their detailed biological examinations.
\end{abstract}

Keywords: graphene oxide; reduced graphene oxide; chitosan; lactic acid; acetic acid; tannic acid; hydroxyapatite

\section{Introduction}

A graphene, two-dimensional, one atom thick, sheet of carbon is a fascinating nanomaterial that has gained a lot of research attention recently, as graphene and its derivatives can be used in many different applications, including those for biomedical purposes [1-3]. This is also due to the fact that they can be chemically or structurally modified to alter or adjust their properties to specific needs $[4,5]$. Graphene oxide (GO), unlike graphene, has functional groups, e.g., carboxylic acid, epoxide, and hydroxyl groups, attached to a carbon sheet. Thermal, chemical or UV treatment of GO results in the formation of reduced graphene oxide $(\mathrm{rGO})$ with a decreased number of oxygen groups and increased electrical conductivity [6]. rGO can be further exploited as a modifier of a polymer matrix to form nanocomposites with favorable properties [5,7-10]. Due to the unique properties, graphene and its derivatives have been widely tested in many applications, especially in biomedicine, as drug carrier materials and scaffolds [11,12]. Many research groups studied the toxicity of GO and rGO using different types of cells [13-15]. The mechanism of interaction of graphene derivatives with cells is still not fully understood but in the case of GO, its toxicity is determined by many factors, including the amount and type of oxygen-containing functional groups, as well as the size, charge, and agglomeration of the sheets [16]. It should be noted that in the case of rGO, an additional factor 
appears, i.e., the type of reducing agent used. The most popular reducing agent for GO is hydrazine [13], but due to its high toxicity, it cannot be used in biomedical applications. Furthermore, the studies showed that $\mathrm{rGO}$ fabricated using $\mathrm{Na}_{2} \mathrm{~S}$ was more toxic than $\mathrm{GO}$, although another research revealed opposite results [17]. For this reason, L-ascorbic acid [18] and natural-based GO reducing agents from plants $[9,19,20]$ and mushrooms [21] have been proposed as an alternative. It is generally accepted that substances of natural origin perform better in biomaterial-related applications.

Polymer matrices used to fabricate nanocomposites can have different origins, but in terms of biocompatibility, versatility, and similarity to native extracellular matrix (ECM) components, natural polymeric materials, such as polysaccharides, are the most attractive class [22]. Chitosan is a wonderful example of a linear, semicrystalline polyaminosaccharide. This biopolymer is a derivative of chitin, the main component of exoskeletons of crustaceans, fungi, and insects, and is characterized by the degree of deacetylation (DD), i.e., the ratio of its two main units, D-glucosamine and N-acetyl-D-glucosamine [23-25]. Both DD and the molar mass of chitosan, together with the presence of amino and hydroxyl groups, affect its physicochemical and biological properties. As a biomaterial, it is biodegradable and biocompatible, moreover, it exhibits analgesic, antibacterial, antifungal, hemostatic, and mucoadhesive properties [25,26]. However, being a natural polymer, chitosan lacks the appropriate mechanical strength for more demanding applications, such as bone tissue engineering. The fabrication of chitosan-based nanocomposites with carefully selected modifying phases can be a solution here.

Bone tissue engineering requires the scaffolds to be not only biocompatible, biodegradable, and preferably bioactive, but also to withstand forces higher than in the case of other tissues. Graphene family materials (GFM) homogeneously dispersed in the polymer matrix to form composites can improve their mechanical strength and also promote cell proliferation and differentiation, accelerating the regeneration of a bone defect [27]. However, when designing such composite materials factors, such as the GFM concentration and arrangement in a polymer matrix, interactions between the matrix, modifying phases, and other components should be carefully considered.

Previously [9], we have tested different green reducing agents for GO and shown that L-ascorbic acid is the most effective. In this study, hybrid nanocomposites based on chitosan and reduced graphene oxide were fabricated for potential use in bone tissue engineering. The CS/rGO hydrogels were formed either in acetic acid or lactic acid-based systems to evaluate the influence of the chosen solvent on the interaction between rGO sheets and the polymer chains, as well as the final properties of the hybrid materials crosslinked with a natural agent—-tannic acid (TAc).

\section{Materials and Methods}

\subsection{Materials}

Chitosan (CS) with a deacetylation degree $>90 \%$ and high molecular weight $(\mathrm{M}=60000-800000 \mathrm{~g} / \mathrm{mol})$ was purchased from Acros-Organics, Morris Plains, NJ, USA. Hydroxyapatite (HA) was purchased from Chema-Elektromet, Rzeszów, Poland. L-ascorbic acid (L-AA), concentrated acetic acid (AAc, $\geq 95,5 \%$ ), lactic acid (LAc, $88 \%)$, tannic acid (TAc), $0.1 \mathrm{M}$ sodium hydroxide $(\mathrm{NaOH})$, hydrochloric acid $(\mathrm{HCl})$ solutions and reagents needed for the preparation of simulated body fluid (SBF), and phosphate-buffered saline (PBS) solutions were obtained from Avantor Performance Materials Poland S.A., Gliwice, Poland. All chemicals were of analytical grade.

\subsection{Graphene Oxide (GO) and Reduced Graphene Oxide (rGO)}

Graphene oxide (GO) was received from the Institute of Electronic Materials Technology (ITME), Poland and synthesized as described in Reference [9]. For reduction, an aqueous suspension of GO $(0.01 \mathrm{mg} / \mathrm{mL})$ was obtained by ultrasonication for $2 \mathrm{~h}$. Next, $300 \mathrm{mg}$ of L-AA was added to $300 \mathrm{~mL}$ of the dispersion and sonicated for another hour. The reduction process of GO was carried out in alkaline conditions. The $\mathrm{pH}$ of the solution was adjusted to 10 (by addition of $0.1 \mathrm{M} \mathrm{NaOH}$ ) to provide colloidal 
stability of GO sheets through electrostatic repulsion forces. Next, the suspension was heated up to $70{ }^{\circ} \mathrm{C}$ and kept for $2 \mathrm{~h}$ under vigorous stirring. The color of the mixture changed from brown to black with the reduction progressing. Obtained rGO suspension was centrifuged, washed a few times with distilled water to remove excess L-AA, then froze $\left(-80^{\circ} \mathrm{C}, 24 \mathrm{~h}\right)$, and freeze-dried $(72 \mathrm{~h}$, Labconco FreeZone).

\subsection{Fabrication of Chitosan/rGO Composites}

The chitosan-based hydrogels were synthesized by simultaneous reduction of GO and self-assembling of the nanocomposites components. In the first step, GO dispersion was prepared by sonicating $37.5 \mathrm{mg}$ of GO powder for $2 \mathrm{~h}$ in $12 \mathrm{~mL}$ of distilled water with $\mathrm{NaOH}$ addition (pH 9-11). Next, $0.375 \mathrm{~g}$ of L-AA was added to the GO dispersion (GO/L-AA ratio was the same as during the synthesis of rGO powder) and the solution was heated up to $70{ }^{\circ} \mathrm{C}$ with continuous stirring for $2 \mathrm{~h}$ to start the reduction process. After that, the dispersion was added to CS solution $(2.5 \mathrm{~g}$ in $38 \mathrm{~mL}$ of $5 \%$ AAc or LAc) containing $0.15 \mathrm{~g}$ of HA. Next, $0.25 \mathrm{~g}$ of TAc was added and the whole system was left on a magnetic stirrer for $24 \mathrm{~h}$ at $40{ }^{\circ} \mathrm{C}$ to allow the further reduction of rGO and self-assembling of hydrogel's components. The mass ratio of CS to modifiers was 2.0 to $0.03,2.0$ to 0.2 , and 2.0 to 0.12 for rGO, Tac, and HA, respectively. The composition of films was selected to obtain optimal physicochemical properties. The preparation of the solution containing more than $1.5 \% \mathrm{wt}$. of rGO was difficult due to the high viscosity. Finally, the mixture was cast onto Teflon dishes and left at room temperature. Series of composites were prepared by changing the type of solvent and mixture ratio. Nanocomposites without HA and/or TAc addition were also synthesized.

\subsection{Characterization}

\subsubsection{X-ray Photoelectron Spectroscopy (XPS)}

X-ray photoelectron spectrometer (Vacuum Systems Workshop Ltd., Crowborough, East Sussex, United Kingdom) with $\mathrm{Mg}$ anode (1253.6 eV K $\alpha$ radiation; $200 \mathrm{~W}$ X-ray excitation source; $3 \times 10^{-8} \mathrm{mbar}$ vacuum; $15^{\circ}$ electron takeoff angle) in the constant analyzer energy mode ( $22 \mathrm{eV}$ pass energy) was used for characterization of GO and rGO.

\subsubsection{X-ray Diffractometry (XRD)}

$X$-ray diffraction measurement of nanofillers and chitosan-based composites was performed using $X^{\prime}$ Pert Pro diffractometer (Malvern Panalytical, Worcestershire, UK) with $\mathrm{Cu}$ K $\alpha$ X-ray sources $(\lambda=$ $1.5406 \AA$ ). D-spacing of GO and rGO was calculated from the Bragg equation:

$$
n \lambda=2 d \sin (\theta)
$$

where $n$ is a positive integer, $\lambda$ is the wavelength of the indecent wave and $\theta$ is the diffraction angle.

\subsubsection{Attenuated Total Reflection Fourier-Transform Infrared Spectroscopy (ATR-FTIR)}

The chemical compositions of materials were analyzed using attenuated total reflection spectroscopy. The spectra were taken in the range $4000-600 \mathrm{~cm}^{-1}$, at resolution $4 \mathrm{~cm}^{-1}$ using Bruker Tensor 27 (Bruker, Poznań, Poland) equipment with a diamond crystal.

\subsubsection{Mechanical Properties}

The tensile properties of composites were measured using a universal testing machine (Zwick 1435, ZwickRoell GmbH \& Co. KG, Ulm, Germany) with a $5 \mathrm{kN}$ load cell, at a constant speed of $1 \mathrm{~mm} / \mathrm{min}$, following ASTM D 882 standard. The reported results were an average of at least five independent measurements for each type of composite. 


\subsubsection{In Vitro Degradation}

In vitro degradation of composites was carried out in PBS solution at $37^{\circ} \mathrm{C}$. Samples were immersed in $15 \mathrm{~mL}$ of PBS solution and stored in an incubator for 6 weeks. Once a week, pH of PBS solution was measured and the medium was renewed. The weight loss was quantified as the change in the dried sample weight over time:

$$
\text { Weight loss }=\frac{\left(W_{0}-W_{t}\right)}{W_{0}} 100 \%
$$

where $W_{0}$ is the initial weight of the sample and $W_{t}$ is the weight after degradation time.

\subsubsection{Scanning Electron Microscopy (SEM)}

The morphology of the prepared composites was characterized by scanning electron microscopy (Nova NanoSEM 200, FEI, Eindhoven, Netherlands) with an accelerating voltage of 10 and $18 \mathrm{kV}$. Chemical compositions of nanocomposites after incubation in SBF was studied by EDS (FEI, Eindhoven, Netherlands) measurements.

\subsubsection{In Vitro Bioactivity Test}

Preliminary bioactivity test was performed using the SBF solution prepared according to the improved protocol described by Bohner [28]. The samples were placed in containers and SBF was added to each of them with constant material surface area $\left(\mathrm{mm}^{2}\right)$ /liquid volume $(\mathrm{mL})$ ratio $(10: 1)$. Containers were placed in an incubator at $37^{\circ} \mathrm{C}$ for 1 and 2 weeks. After incubation, samples were rinsed with distilled water and dried at $37^{\circ} \mathrm{C}$ for subsequent analyses.

\subsubsection{Wettability}

The wettability of the composite films was assessed by water contact angle (WCA) measurement using goniometer (Drop Shape Analyzer, KRÜSS GmbH, Hamburg, Germany) at room temperature. The sessile drop of deionized water was deposited on the surface and contact angle was calculated. For each type of material, six drops were measured and the average value was reported.

\subsubsection{Determination of the Number of Free Amino Groups}

The number of free amino groups of chitosan-based composites was determined by ninhydrin assay [29] to evaluate the cross-linking effect of rGO, HA, and TAc. Briefly, $1.5 \mathrm{mg}$ of freeze-dried sample was heated up with $1 \mathrm{~mL}$ of ninhydrin solution in a water bath for $1 \mathrm{~h}$. After cooling down to room temperature, the obtained solution was diluted in $5 \mathrm{~mL}$ of isopropanol/water (1:1) mixture. The optical absorbance was recorded with a UV-vis spectrophotometer (CE2502, Cecil Instruments Ltd., Cambridge, United Kingdom) at $570 \mathrm{~nm}$. Various glycine concentrations were used to obtain a calibration curve. The number of free amino groups in the tested samples was proportional to the optical absorbance of the obtained solution and was calculated as:

$$
\text { Amount of free amino groups }=100 \%-\left(\left(\frac{\left(C_{p}-C_{c}\right)}{C_{p}}\right) 100 \%\right)
$$

where: $C_{p}$ - concentration of free $\mathrm{NH}_{2}$ groups in pristine chitosan; $C_{c}$-concentration of free $\mathrm{NH}_{2}$ groups in composites.

\subsubsection{Cytocompatibility}

The CS composite solutions were cast into a 48 -well plate and dried overnight at $37^{\circ} \mathrm{C}$. Next, samples were incubated in $0.5 \mathrm{M} \mathrm{NaOH}$ solution for $4 \mathrm{~h}$. After removing $\mathrm{NaOH}$, samples were washed with distilled water three times for $10 \mathrm{~min}$ and dried overnight at $37^{\circ} \mathrm{C}$. Samples were sterilized 
by incubating with $70 \%$ ethanol at room temperature for $30 \mathrm{~min}$ and subsequently dried for $1 \mathrm{~h}$ at $37^{\circ} \mathrm{C}$. Cytocompatibility of the as-prepared samples was evaluated in a direct contact with MG-63 cells (European Collection of Cell Cultures, Salisbury, UK) cultured in Eagle's minimal essential medium (EMEM, PAN BIOTECH, Aidenbach, Germany) supplemented with $10 \%$ fetal bovine serum, $1 \%$ penicillin-streptomycin, and $0.1 \%$ sodium pyruvate (all of them from PAA Laboratories Gmbh, Pasching, Oberosterreich, Austria). Tissue culture polystyrene (TCPS) served as a control. The cells $\left(1.5 \times 10^{4}\right.$ cells $\left./ \mathrm{cm}^{2}\right)$ were grown on the tested materials for $24 \mathrm{~h}, 3$ days, and 7 days. Their metabolic activity was measured via Alamar Blue assay [30]. Three samples of each type were tested. Briefly, $0.5 \mathrm{~mL}$ of reagent $(10 \%(\mathrm{w} / \mathrm{v})$ Resazurin solution in PBS, Sigma-Aldrich) was added and incubated with cells for $4 \mathrm{~h}$ at $37^{\circ} \mathrm{C}$. After $4 \mathrm{~h} 100 \mu \mathrm{L}$ from each sample were transferred to a black 96-well plate and resazurin reduction was measured via fluorescence (kex.-530 nm, kem. - $590 \mathrm{~nm}$; FLUOstar Omega, BMG Labtech, Ortenberg, Germany). The results are shown as a mean \pm standard deviation.

\section{Results}

\subsection{Chemical Reduction of GO}

All reagents used for the synthesis of nanocomposite hydrogels were characterized using ATR and XRD method (Figure 1a,b). Successful removal of oxygen-containing groups from GO was also confirmed using XPS (Figure 1c,d).
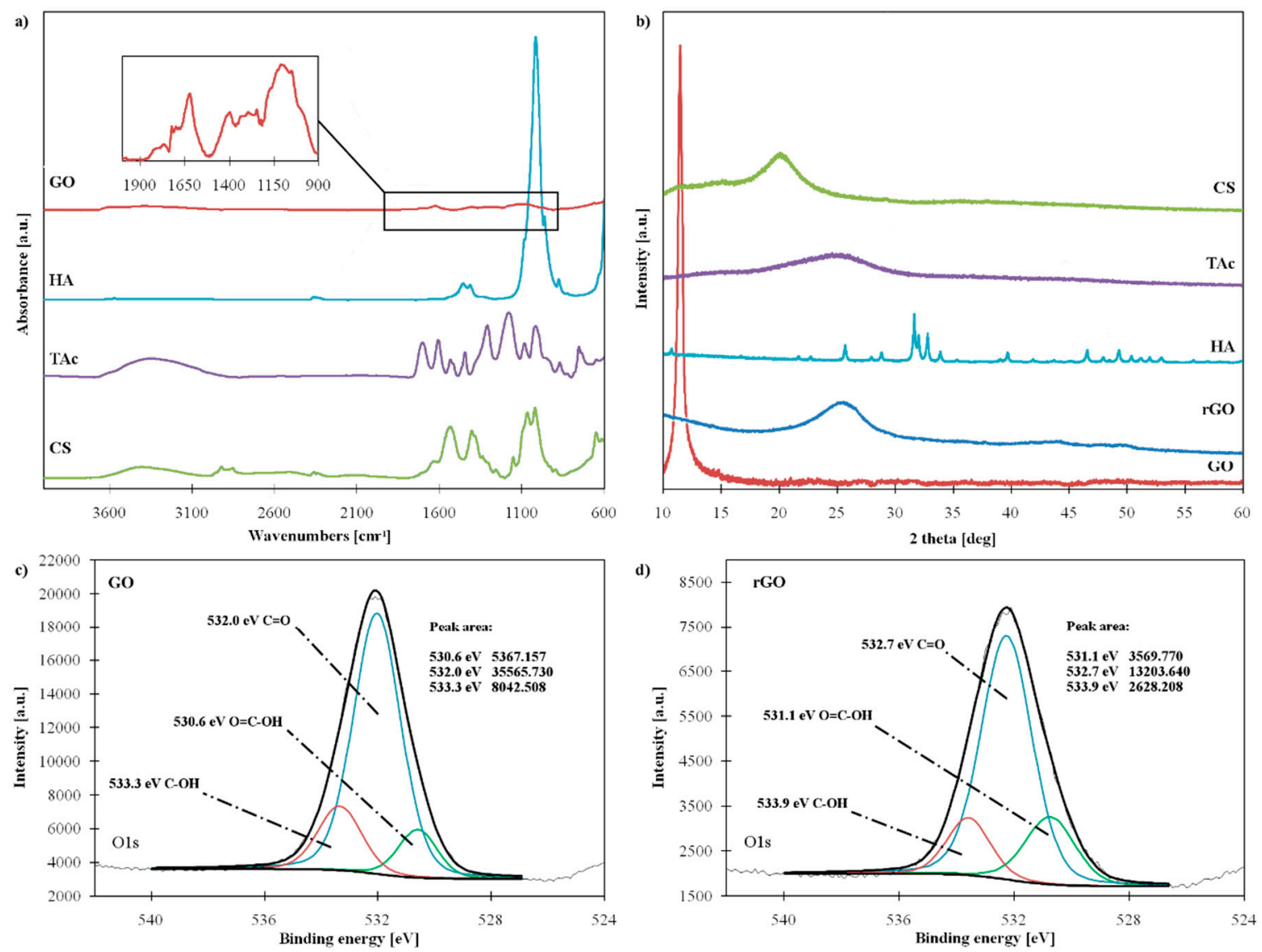

Figure 1. (a) ATR-FTIR (Attenuated Total Reflection Fourier-Transform Infrared) spectra, (b) XRD (X-ray Diffractometry) patterns of materials used for chitosan (CS) nanocomposites synthesis, and XPS (X-ray Photoelectron Spectroscopy) spectra of (c) graphene oxide (GO) and (d) reduced graphene oxide (rGO); (HA-hydroxyapatite, TAc—-tannic acid). 
ATR spectrum of GO provides information about oxygen functionalities attached to the GO surface. The broad peak at $3387 \mathrm{~cm}^{-1}$ corresponded to -OH stretching vibrations. The peak of skeletal vibration from not oxidized $\mathrm{C}=\mathrm{C}$ bond showed at $1620 \mathrm{~cm}^{-1}$. The characteristics peaks assigned to various types of oxygen-groups: $\mathrm{C}=\mathrm{O}$ (stretching vibrations), $\mathrm{C}-\mathrm{O}$ (stretching vibrations of $\mathrm{C}-\mathrm{O}-\mathrm{C}$ ) and $\mathrm{C}-\mathrm{O}$ (stretching vibrations of $\mathrm{C}-\mathrm{OH}$ groups) can be found at $1723 \mathrm{~cm}^{-1}, 1771 \mathrm{~cm}^{-1}$, and $1397 \mathrm{~cm}^{-1}$, respectively $[18,31]$. After the reduction process, the intensity of these groups was significantly reduced, which confirmed the successful removal of oxygen-containing groups from GO.

Changes in the chemical compositions of GO were also examined by XPS. High-resolution O1s spectrum of GO (Figure 1c) showed three peaks assigned to different types of oxygen components: Oxygen of $\mathrm{C}-\mathrm{O}(533.3 \mathrm{eV})$, oxygen of $\mathrm{C}=\mathrm{O}(532.0 \mathrm{eV})$ and oxygen of $\mathrm{O}=\mathrm{C}-\mathrm{OH}(530.6 \mathrm{eV})[21,31]$. In the O1s spectrum of rGO, the same peaks can be observed but their intensity is significantly smaller compared to GO, as shown in Figure 1d. The oxygen peak area of rGO decreased by $63 \%$.

The GO and rGO were also characterized by XRD. GO presented a sharp, single peak at $2 \theta$ value $11.51^{\circ}$ corresponding to an interlayer d-spacing $0.77 \mathrm{~nm}$. In contrast, in the rGO pattern the typical GO peak disappeared and a new wide peak appeared at 25.70 (d-spacing $0.35 \mathrm{~nm}$ ), as presented in Figure $1 \mathrm{~b}$. After reduction, the interlayer distance between the sheets significantly decreased as a result of the removal of oxygen-containing functional groups [32,33].

\subsection{Characterization of Nanocomposite Hydrogels}

To characterize components of the hydrogels, ATR and XRD measurements were done (Figure 1a,b). As previously demonstrated, GO contains oxygen-functional groups attached to the surface, which can potentially interact with chitosan groups. The characteristic peaks of CS in ATR spectra appeared at $1639 \mathrm{~cm}^{-1}, 1534 \mathrm{~cm}^{-1}$, and $1402 \mathrm{~cm}^{-1}$ which corresponded to the $\mathrm{C}=\mathrm{O}$ in the amide I (stretching vibrations in -NHCO-), $\mathrm{N}-\mathrm{H}$ vibrations in $\mathrm{NH}_{2}$ group and $\mathrm{CH}_{3}$ deformations in amide groups, respectively. Peaks at $1153 \mathrm{~cm}^{-1}$ and $1020 \mathrm{~cm}^{-1}$ were attributed to asymmetric stretching in C-O-C bridge and stretching vibrations in the C-O group [34,35]. The characteristic peak of HA at $1015 \mathrm{~cm}^{-1}$ corresponded to P-O vibrations [36]. The spectrum of TAc reflects its complex structure. Peaks located at $1702 \mathrm{~cm}^{-1}, 1443 \mathrm{~cm}^{-1}, 1177 \mathrm{~cm}^{-1}$, and $753 \mathrm{~cm}^{-1}$ were attributed to the $\mathrm{C}=\mathrm{O},-\mathrm{C}-\mathrm{C}_{\text {aromatic }}$, $\mathrm{C}-\mathrm{O}$ stretching vibrations, and $\mathrm{C}=\mathrm{C}$ distortion vibrations in benzene rings (32). The spectra of $\mathrm{CS}$ composites in AAC without TAc (CS/rGO and CS/rGO/HA) looked similar to the spectrum of pristine chitosan, as shown in Figure 2a. Characteristic peaks of GO and HA were not present, confirming the successful reduction of GO and good dispersion of the fillers in the matrix. The introduction of TAc significantly altered the spectra (CS/rGO/TAc and $\mathrm{CS} / \mathrm{rGO} / \mathrm{HA} / \mathrm{TAc})$. The smaller intensity of the peak corresponding to $\mathrm{N}-\mathrm{H}$ band in $-\mathrm{NH}_{2}$ and shift from $1534 \mathrm{~cm}^{-1}$ to $1550 \mathrm{~cm}^{-1}$ can be related to the deformation of $\mathrm{NH}^{3+}$. Interaction of CS and TAc under acidic conditions caused the ionization of amine groups [37]. In CS/rGO/TAc spectra, new peaks showed up at $1639 \mathrm{~cm}^{-1}, 1556 \mathrm{~cm}^{-1}$ and $1257 \mathrm{~cm}^{-1}$, which might be due to the interaction between components of composite and creating new amide (I, II and III) bonds. The ATR-FTIR spectra of the hydrogels prepared in lactic acid (Figure 2b) reveal some changes compared to the acetic acid systems. As seen in Figure 2b, the peaks corresponding to $-\mathrm{CH}_{3}$ deformation in amide groups at $1402 \mathrm{~cm}^{-1}$ and $1371 \mathrm{~cm}^{-1}$ decreased significantly. Moreover, the FTIR spectra of all the LAc samples show a large band at $1568 \mathrm{~cm}^{-1}$ attributed to $-\mathrm{NH}_{2}$. This shift of the amine group vibration to higher wavelength can be associated with the formation of a carboxylate between protonated $-\mathrm{NH}^{3+}$ of chitosan and $-\mathrm{COO}^{-}$groups of LAc. The peak at $1721 \mathrm{~cm}^{-1}$ can be attributed to - $\mathrm{COOH}$ groups and suggest that free lactic acid is still present in the samples [38]. The FTIR results suggest that CS and LAc may interact with each other forming chitosan salt, i.e., chitosan lactate. 

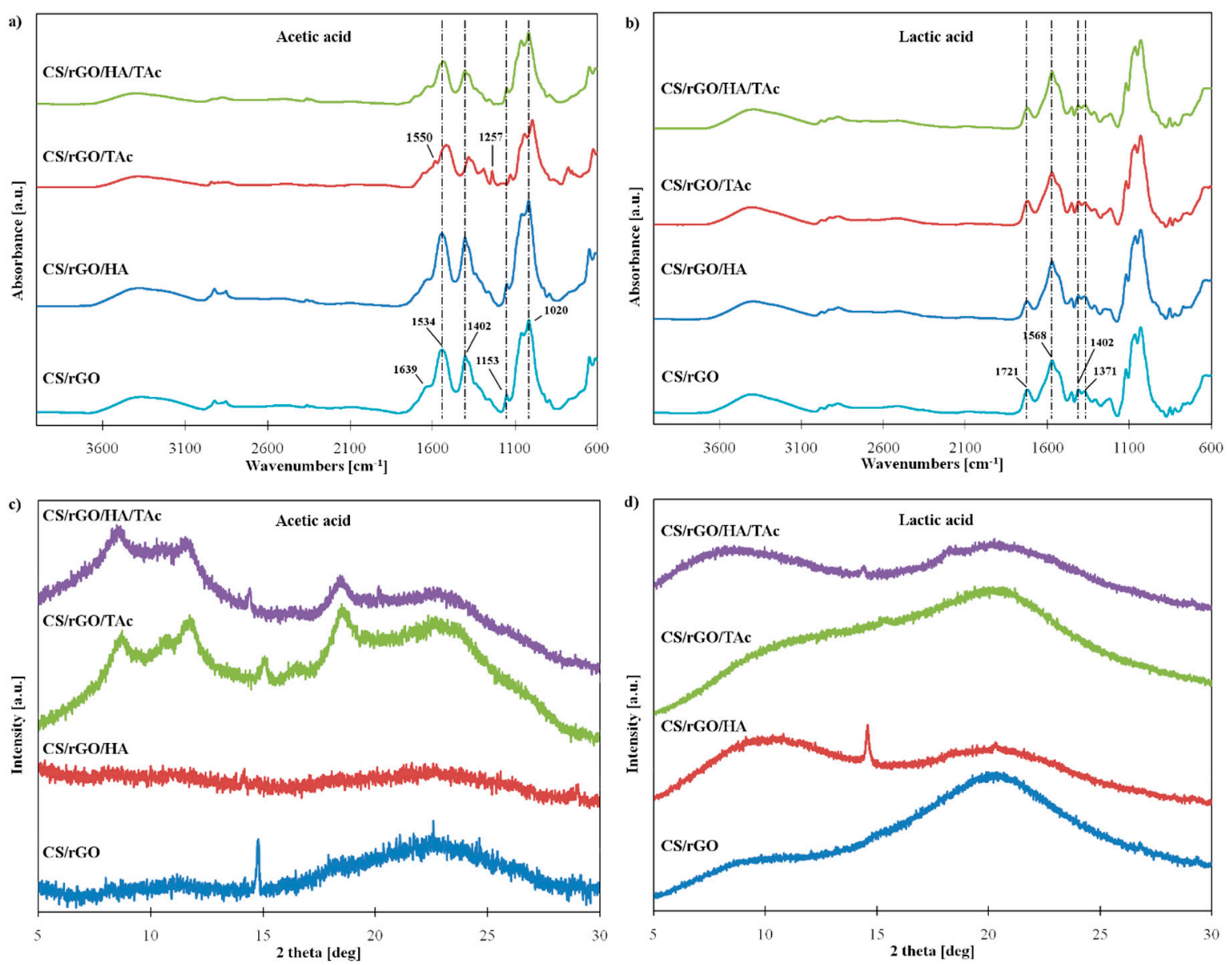

Figure 2. (a,b) ATR-FTIR (Attenuated Total Reflection Fourier-Transform Infrared) spectra and (c,d) XRD (X-ray Diffractometry) patterns of composite samples obtained from $(\mathbf{a}, \mathbf{c})$ acetic acid-based and $(\mathbf{b}, \mathbf{d})$ lactic acid-based solvent system (CS-chitosan, rGO-reduced graphene oxide, HA-hydroxyapatite, TAc-tannic acid).

The crystallinity of polymer is a very important factor, which determines its mechanical properties, as well as stability and biological response. CS can have three forms-amorphous, hydrated crystalline, and anhydrous crystalline. The pristine CS had a fully amorphous character, what can be identified by a broad peak centered at $20.07^{\circ}$ (Figure 1 b). The composite prepared by dissolving CS in AAc without TAc addition exhibited similar behavior, as shown in Figure 2c. The sharp peak at $14.81^{\circ}$ in the $\mathrm{CS} / \mathrm{rGO}$ pattern is the result of the anhydrous crystalline structure in the CS matrix. The composites with TAc (CS/rGO/TAc and $\mathrm{CS} / \mathrm{rGO} / \mathrm{HA} / \mathrm{TAc}$ ) showed a significant rise in the peaks assigned to crystalline forms of CS. Several peaks at $\approx 8,11,12^{\circ}$ (hydrous forms) and $\approx 15,18^{\circ}$ (anhydrous forms) were found [39]. This phenomenon can be attributed to the synergistic effect of rGO and TAc as cross-linkers. rGO can support nucleation through exfoliation in alkaline conditions, what is connected with the increased surface area and strong electrostatic interaction with the polymer chains. All patterns of LAc-based composites exhibited an amorphous or almost fully amorphous character, even with TAc addition, as shown in Figure $2 \mathrm{~d}$. The single sharp peak at $\approx 15^{\circ}$ corresponding to the anhydrous form of chitosan appeared in CS/rGO/HA (LAc) spectra. We speculate that the interaction between CS and LAc affected the packing of CS chains and resulted in amorphous forms of the composites. Peaks of rGO and HA did not appear in XRD patterns of composites, which confirmed good dispersion of fillers and strong interaction between CS and rGO.

Interaction between the components of the nanocomposites and amino functional groups of CS was also examined by ninhydrin assay (Figure 3). The addition of $1.5 \%(\mathrm{w} / \mathrm{w})$ of rGO decreased the number of free amino groups in CS chains by more than $40 \%$, confirming the reaction between 
functional groups attached to rGO surface and CS. Interestingly, the TAc addition reduced this effect. This allows to speculate that TAc interacted with rGO as well and impeded the cross-linking effect of rGO. A similar trend was observed for composite prepared in LAc, but the amount of free amino groups was smaller compared to AAc. This is consistent with ATR and XRD results suggesting an interaction between CS and LAc.

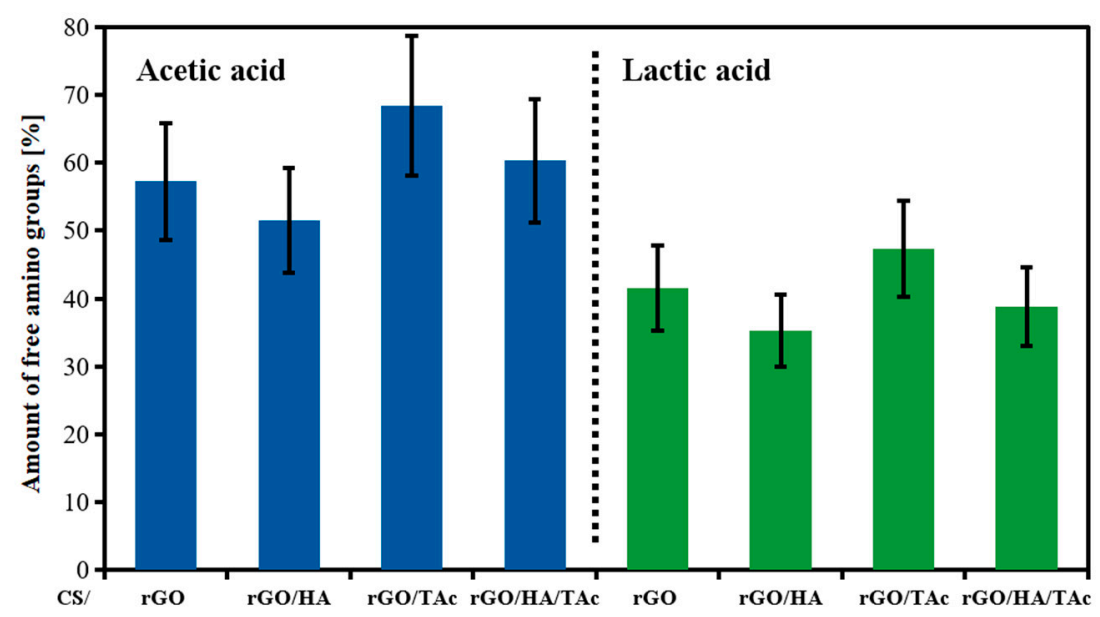

Figure 3. Amount of free amino groups in CS composites measured by ninhydrin assay (CS—chitosan, rGO—reduced graphene oxide, HA—hydroxyapatite, TAc—-tannic acid).

The effect of rGO and TAc on the degradation rate of CS in PBS medium was also investigated. Pristine chitosan sample dissolved completely after one day. The composites prepared in LAc solution dissolved after one week, except for $\mathrm{CS} / \mathrm{rGO} / \mathrm{HA} / \mathrm{TAc}$. Figure 4a shows the change in $\mathrm{pH}$ of PBS solution during six weeks of degradation in $37^{\circ} \mathrm{C}$. In the case of $\mathrm{CS} / \mathrm{rGO}$ (AAc), the $\mathrm{pH}$ value decrease was the least significant. After the first week, the $\mathrm{pH}$ decreased from 7.4 to 6.5. This is due to the removal of AAc from the sample. In the next step, the $\mathrm{pH}$ of medium returned to neutral value and the pattern plateaued. The degradation behavior of other samples prepared in AAc was similar but the decrease of $\mathrm{pH}$ value in the first week was more significant. In the case of $\mathrm{CS} / \mathrm{rGO} / \mathrm{HA} / \mathrm{TAc}$ (LAc), change was more rapid and $\mathrm{pH}$ decreased to 4.1 in the first week. The degradation was also analyzed by measuring the weight loss of dried samples (Figure $4 \mathrm{~b}$ ). In the first step, the degradation rate was rapid for all composites and in the next, it was relatively smaller. The patterns plateaued after two weeks. The kinetics of degradation was the slowest for CS/rGO (AAc) and CS/rGO/HA (AAc). The weight loss after six weeks of degradation was $\approx 40 \%$, while for $\mathrm{CS} / \mathrm{rGO} / \mathrm{TAc}$ (AAc) and $\mathrm{CS} / \mathrm{rGO} / \mathrm{HA} / \mathrm{TAc}$ ( $\mathrm{AAc}$ ) it was $\approx 90 \%$ and $\approx 80 \%$, respectively. The increased stability of $\mathrm{CS} / \mathrm{rGO}$ (AAc) and $\mathrm{CS} / \mathrm{rGO} / \mathrm{HA}$ (AAc) can be explained by the presence of well-exfoliated rGO nanofillers in the polymer matrix. The mobility of the CS chains was limited by absorption onto the rGO surface through hydrogen bonding. In addition, rGO can act as a barrier for ions from the PBS solution. A faster degradation of the composites with TAc addition can result from limited interactions between CS and rGO, as concluded from the ninhydrin assay. The weight loss of CS/rGO/HA/TAc (LAc) was $\approx 95 \%$. This can be related to the interaction between CS and LAc, which resulted in the formation of more soluble forms of CS. Other composites prepared in LAc dissolved completely after one week.

The mechanical behavior of the nanocomposite films was investigated by tensile test. CS/rGO (AAc) and CS/rGO/HA (AAc) were soft and easy to bend without cracking. The addition of TAc caused an increase in the degree of crystallinity and hence the fragility of the composites in a dry state. $\mathrm{CS} / \mathrm{rGO}(\mathrm{AAc}$ ) and $\mathrm{CS} / \mathrm{rGO} / \mathrm{HA}$ (AAc) films showed balanced mechanical properties (Figure 5). The composites prepared in LAc solution behaved differently, what is believed to be related to their amorphous structure. The elongation at break of CS/rGO (LAc) exceeded $50 \%$. At the same time, Young's modulus of the film synthesized in LAc increased even by $50 \%$ compared to AAc-based composites. The positive effect of TAc was also noticeable. 
a)

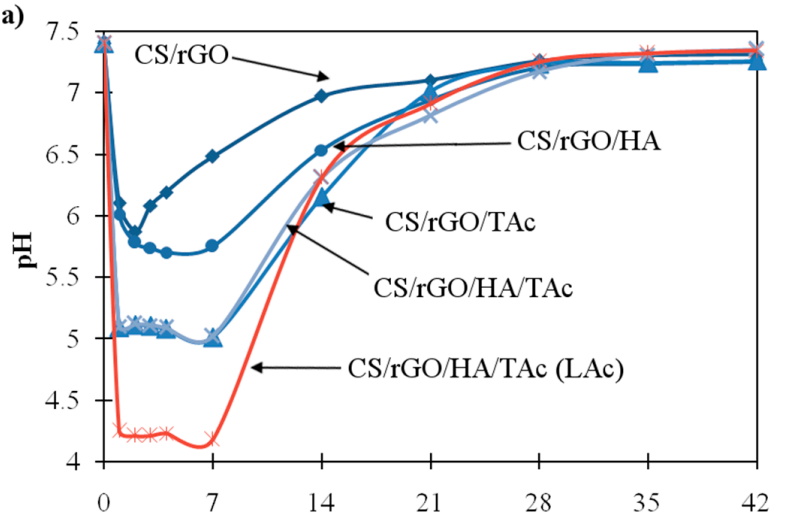

b)

Degradation time [days]

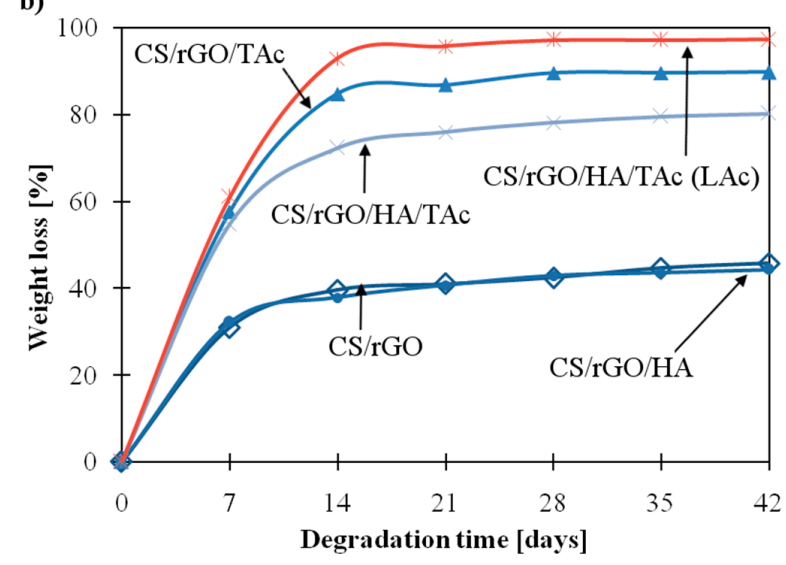

Figure 4. Degradation behavior of CS nanocomposites in PBS (Phosphate Buffered Saline) solution: (a) Changes of $\mathrm{pH}$ value of degradation media, (b) weight loss of samples during incubation, at $37^{\circ} \mathrm{C}$. Blue curves - composites prepared in AAc (acetic acid), red curve-composite prepared in LAc (lactic acid); (CS—chitosan, rGO—reduced graphene oxide, HA-hydroxyapatite, TAc—tannic acid).

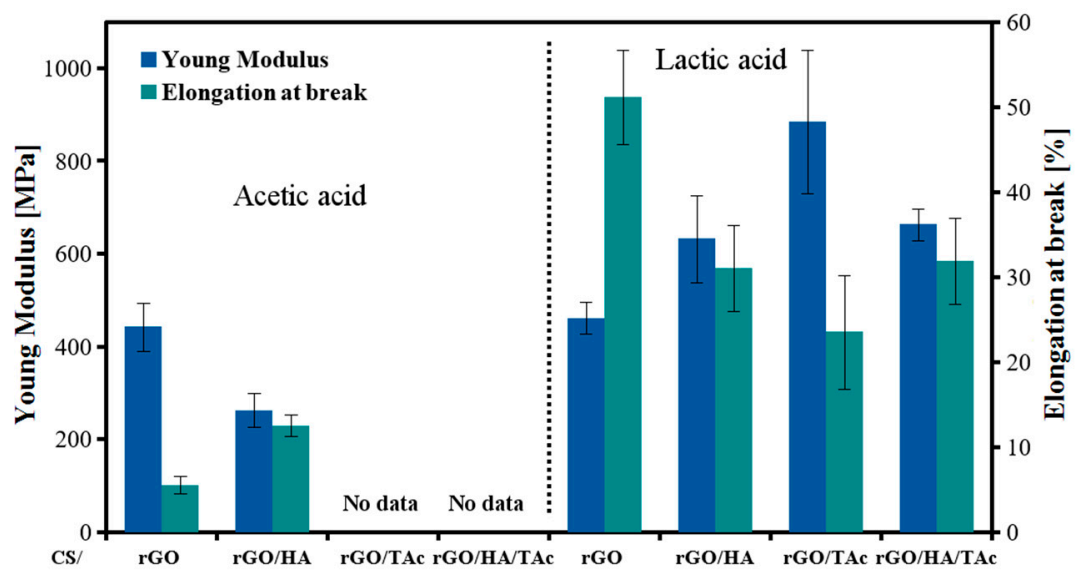

Figure 5. Young's modulus and elongation at break of CS nanocomposites (CS—chitosan, rGO—reduced graphene oxide, HA-hydroxyapatite, TAc—tannic acid).

Good mechanical properties of the composites are provided by specific, dense microstructure with rGO sheets arranged parallel to each other and the film's surface (Figure 6) and strong $\pi-\pi$ interaction between layers combined with strong interfacial adhesion between CS and rGO.

The surface wettability of nanocomposites is an important factor for tissue engineering. It was investigated with the water contact angle measurement. Due to the hydrophobic nature, the water contact angle (WCA) of pristine CS film was $109.71^{\circ} \pm 2.31^{\circ}$. Introduction of rGO into polymer matrix 
affected WCA significantly, as shown in Figure 6. The character of all samples prepared in the AAc solution changed to hydrophilic. The biggest improvement was observed for $\mathrm{CS} / \mathrm{rGO}$ (AAc) $\left(75.40^{\circ} \pm\right.$ $\left.4.32^{\circ}\right)$. The rough film's surface with arranged parallel—well visible under SEM-large rGO sheets was responsible for WCA improvement. This effect is even more significant in the case of the samples prepared in LAc. The WCA value of CS/rGO (LAc) was $36.71^{\circ} \pm 4.53^{\circ}$.

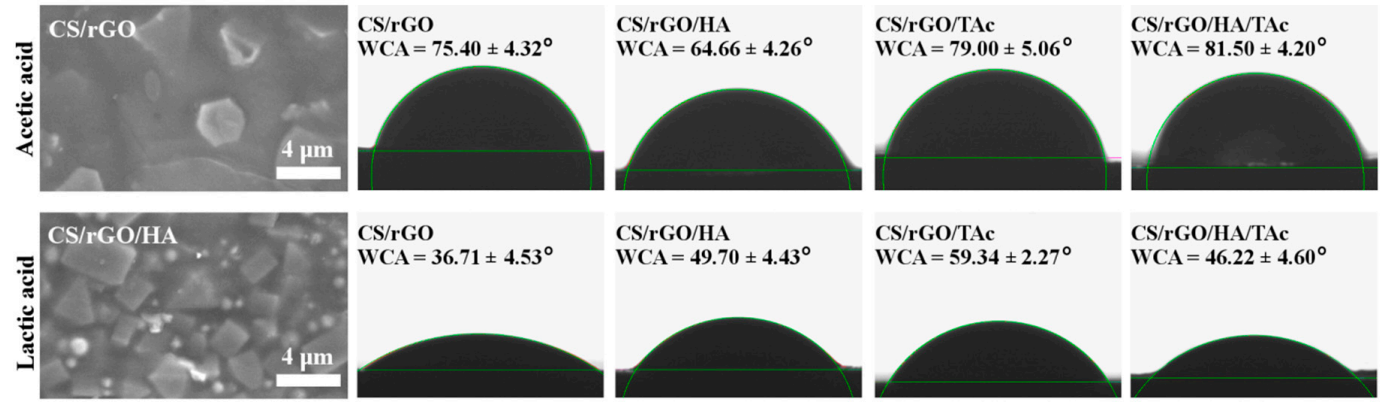

Figure 6. Representative SEM (Scanning Electron Microscopy) images of CS nanocomposites surface (CS/rGO prepared in AAc and CS/rGO/HA prepared in LAc solution) and representative images of water droplets on the samples' surface with mean values of water contact angle-WCA (CS - chitosan, rGO—reduced graphene oxide, HA — hydroxyapatite, TAc — tannic acid, AAc—acetic acid, LAc-lactic acid).

The prepared nanocomposites were immersed in SBF solution to evaluate their in vitro bioactivity. The capacity to bond with living bone by the formation of an apatite interface layer on the material surface is an essential factor in tissue engineering. After 14 days of incubation, isolated groups of calcium phosphate particles were observed on all of the tested samples. SEM images in Figure 7 show that a dense, continuous layer of bone-like apatite was created on the surface of the nanocomposite after 28 days of incubation. Nucleated particles were spherical. $\mathrm{Ca} / \mathrm{P}$ ratio was measured by EDS to investigate the chemical composition of the layers. The analysis confirmed that the $\mathrm{Ca} / \mathrm{P}$ ratio of all of the layers was in the range 1.56-1.86 after 28 days, which is very close to the $\mathrm{Ca} / \mathrm{P}$ ratio of HA. Interestingly, TAc addition resulted in an increase in the value of $\mathrm{Ca} / \mathrm{P}$ ratio. Furthermore, the particles grown on nanocomposites synthesized in LAc had a slightly larger size. Preliminary in vitro tests confirmed cytocompatibility of the fabricated nanocomposites in contact with MG-63 cells (Figure 8).
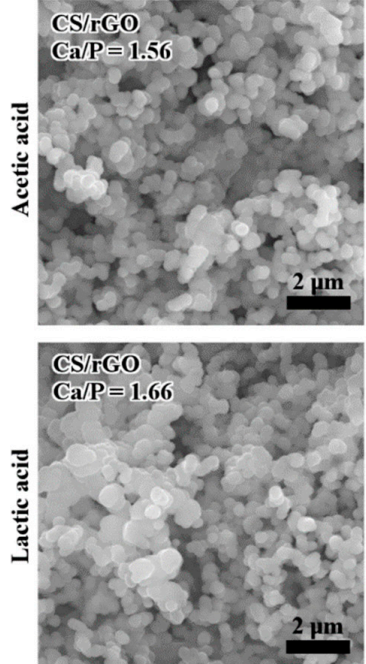
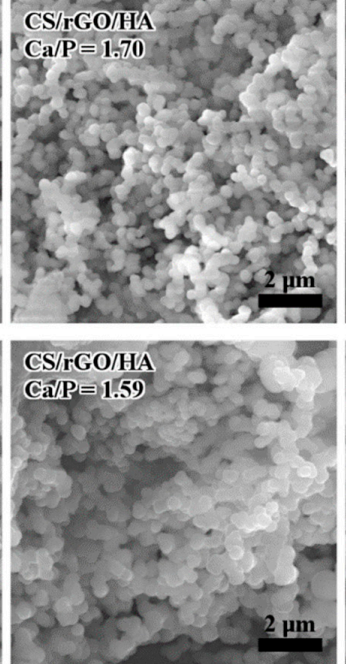
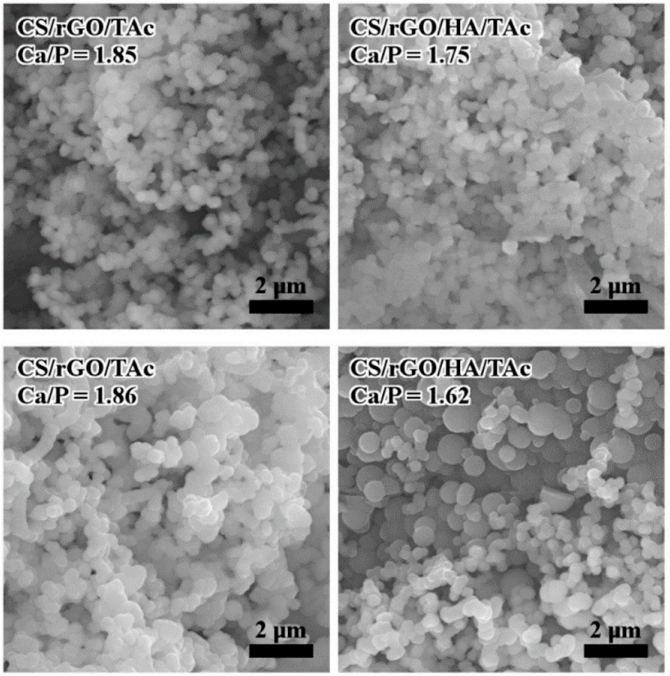

Figure 7. Representative SEM (Scanning Electron Microscopy) images of the surface of CS nanocomposites after 28 days of incubation in SBF solution (CS — chitosan, rGO-reduced graphene oxide, HAhydroxyapatite, TAc-tannic acid). 


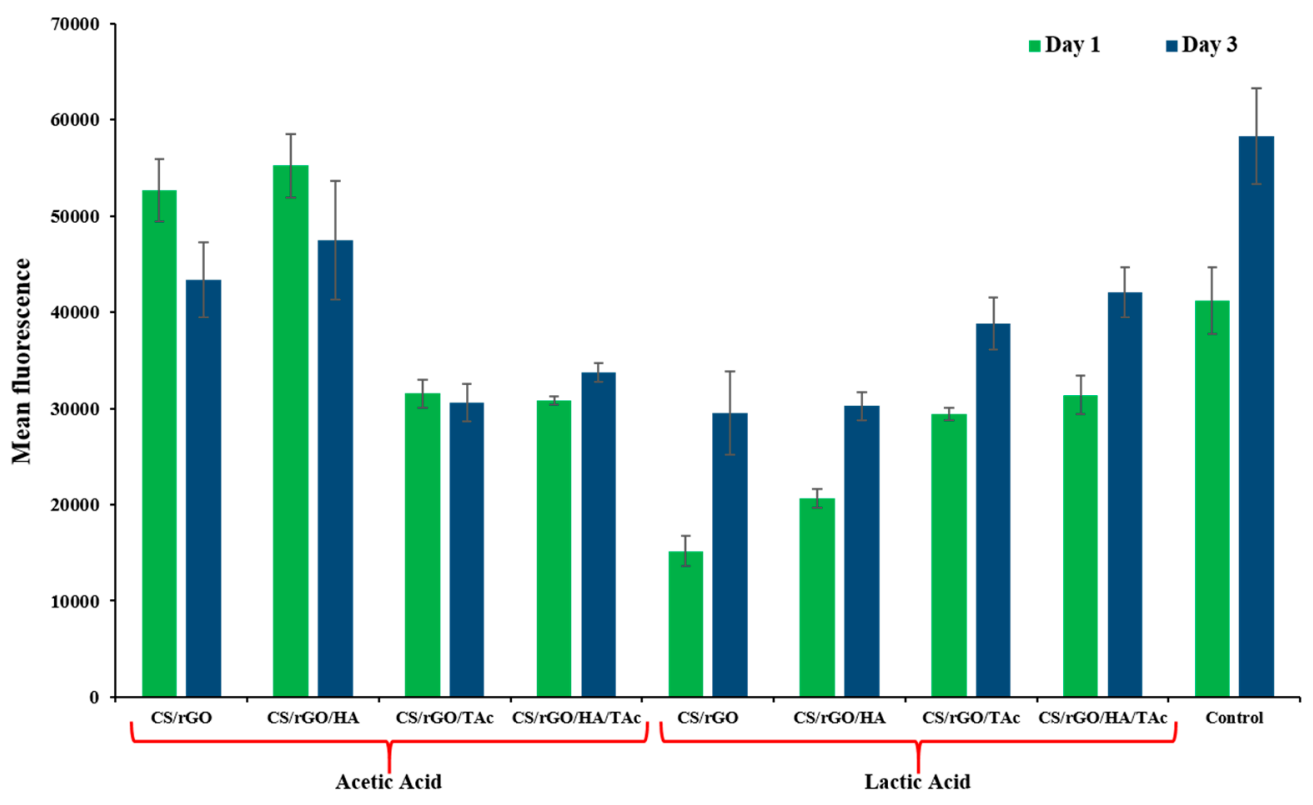

Figure 8. Alamar Blue assay—mean fluorescence after $24 \mathrm{~h}$ and 3 days (CS—chitosan, rGO—reduced graphene oxide, HA-hydroxyapatite, TAc—tannic acid).

\section{Conclusions}

The nanocomposites based on CS and exfoliated rGO were prepared successfully by a simple method with TAc as a crosslinker. Reduction of rGO during composite preparation allowed for the self-assembly of CS and rGO using $\pi-\pi$ interaction between well-exfoliated rGO layers, which stacked in a special orientation, parallel to each other and the sample surface. This positively affected the wettability, mechanical properties, and stability of the composites. Crosslinking by TAc influenced the crystallinity of the composites, increased Young's modulus and decreased the elongation at break. It also turned out that the type of solvent had a significant effect on the properties of the obtained hydrogels. A preliminary, in vitro biological test confirmed the cytocompatibility of the materials. More detailed examinations of the biological performance of the materials will be the next step.

Author Contributions: K.K. performed the synthesis and physicochemical characterization of the materials, visualized data, and wrote the original draft; P.D.-P. directed the studies, interpreted the results, revised, and edited the manuscript; M.K.-B. performed cell assays and interpreted the results, revised the final manuscript; J.C. supervised the studies, acquired funding, and revised the final manuscript. All authors read and approved the final manuscript.

Funding: This research was funded by the National Center for Research and Development, Poland (BioMiStem grant No. STRATEGMED3/303570/7/NCBR/2017).

Acknowledgments: Authors would like to kindly acknowledge Joanna Jagiełło and Ludwika Lipińska from the Institute of Electronics Materials Technology (ITME) in Warsaw for providing graphene oxide and Professor Marek Nocun (Faculty of Materials Science and Ceramics, AGH University of Science and Technology) for his assistance with XPS analysis.

Conflicts of Interest: The authors declare no conflict of interest.

\section{References}

1. Geim, A.K.; Novoselov, K.S. The rise of graphene. Nat. Mater. 2007, 6, 183-191. [CrossRef] [PubMed]

2. Shin, S.R.; Li, Y.-C.; Jang, H.L.; Khoshakhlagh, P.; Akbari, M.; Nasajpour, A.; Zhang, Y.S.; Tamayol, A.; Khademhosseini, A. Graphene-based materials for tissue engineering. Adv. Drug Deliv. Rev. 2016, 105, 255-274. [CrossRef] [PubMed]

3. Chung, C.; Shin, D.; Ryoo, S.-R.; Min, D.-H.; Kim, Y.-K.; Hong, B.H. Biomedical Applications of Graphene and Graphene Oxide. Accounts Chem. Res. 2013, 46, 2211-2224. [CrossRef] [PubMed] 
4. Luo, J.; Kim, J.; Huang, J. Material Processing of Chemically Modified Graphene: Some Challenges and Solutions. Accounts Chem. Res. 2013, 46, 2225-2234. [CrossRef] [PubMed]

5. Yang, K.; Feng, L.; Hong, H.; Cai, W.; Liu, Z. Preparation and functionalization of graphene nanocomposites for biomedical applications. Nat. Protoc. 2013, 8, 2392-2403. [CrossRef] [PubMed]

6. Bianco, A.; Cheng, H.-M.; Enoki, T.; Gogotsi, Y.; Hurt, R.H.; Koratkar, N.; Kyotani, T.; Monthioux, M.; Park, C.R.; Tascón, J.M.; et al. All in the graphene family-A recommended nomenclature for two-dimensional carbon materials. Carbon 2013, 65, 1-6. [CrossRef]

7. Justin, R.; Chen, B. Strong and conductive chitosan-reduced graphene oxide nanocomposites for transdermal drug delivery. J. Mater. Chem. B 2014, 2, 3759. [CrossRef]

8. Kosowska, K.; Domalik-Pyzik, P.; Chłopek, J. Graphene oxide and green-synthesized reduced graphene oxide in chitosan-based nanocomposites. Eng. Biomater. 2017, 142, 11-16.

9. Kosowska, K.; Domalik-Pyzik, P.; Nocuń, M.; Chłopek, J. Chitosan and graphene oxide/reduced graphene oxide hybrid nanocomposites-Evaluation of physicochemical properties. Mater. Chem. Phys. 2018, 216, 28-36. [CrossRef]

10. Wang, L.; Lu, X.; Lei, S.; Song, Y. Graphene-based polyaniline nanocomposites: Preparation, properties and applications. J. Mater. Chem. A 2014, 2, 4491-4509. [CrossRef]

11. Tabish, T.A.; Chabi, S.; Ali, M.; Xia, Y.; Jabeen, F.; Zhang, S. Tracing the Bioavailability of Three-Dimensional Graphene Foam in Biological Tissues. Materials 2017, 10, 336. [CrossRef] [PubMed]

12. Bahrami, S.; Baheiraei, N.; Mohseni, M.; Razavi, M.; Ghaderi, A.; Azizi, B.; Rabiee, N.; Karimi, M. Three-dimensional graphene foam as a conductive scaffold for cardiac tissue engineering. J. Biomater. Appl. 2019, 34, 74-85. [CrossRef] [PubMed]

13. Tabish, T.; Pranjol, M.Z.I.; Hayat, H.; Rahat, A.A.M.; Abdullah, T.M.; Whatmore, J.L.; Zhang, S. In vitro toxic effects of reduced graphene oxide nanosheets on lung cancer cells. Nanotechnology 2017, 28, 504001. [CrossRef] [PubMed]

14. Lategan, K.; Alghadi, H.; Bayati, M.; De Cortalezzi, M.F.; Pool, E. Effects of Graphene Oxide Nanoparticles on the Immune System Biomarkers Produced by RAW 264.7 and Human Whole Blood Cell Cultures. Nanomaterials 2018, 8, 125. [CrossRef]

15. Tabish, T.A.; Zhang, S.; Winyard, P.G. Developing the next generation of graphene-based platforms for cancer therapeutics: The potential role of reactive oxygen species. Redox Biol. 2018, 15, 34-40. [CrossRef]

16. Ou, L.; Song, B.; Liang, H.; Liu, J.; Feng, X.; Deng, B.; Sun, T.; Shao, L. Toxicity of graphene-family nanoparticles: A general review of the origins and mechanisms. Part. Fibre Toxicol. 2016, 13, 57. [CrossRef]

17. Wu, Y.; Wang, F.; Wang, S.; Ma, J.; Xu, M.; Gao, M.; Liu, R.; Chen, W.; Liu, S. Reduction of graphene oxide alters its cyto-compatibility towards primary and immortalized macrophages. Nanoscale 2018, 10, 14637-14650. [CrossRef]

18. Zhang, J.; Yang, H.; Shen, G.; Cheng, P.; Zhang, J.; Guo, S. Reduction of graphene oxide via 1 -ascorbic acid. Chem. Commun. 2010, 46, 1112-1114. [CrossRef]

19. Upadhyay, R.; Soin, N.; Bhattacharya, G.; Saha, S.; Barman, A.; Roy, S. Grape extract assisted green synthesis of reduced graphene oxide for water treatment application. Mater. Lett. 2015, 160, 355-358. [CrossRef]

20. Liu, C.; Liu, H.; Zhang, K.; Dou, M.; Pan, B.; He, X.; Lu, C. Partly reduced graphene oxide aerogels induced by proanthocyanidins for efficient dye removal. Bioresour. Technol. 2019, 282, 148-155. [CrossRef]

21. Muthoosamy, K.; Bai, R.G.; Abubakar, I.B.; Sudheer, S.M.; Lim, H.N.; Loh, H.-S.; Huang, N.M.; Chia, C.H.; Manickam, S.; Hongngee, L.; et al. Exceedingly biocompatible and thin-layered reduced graphene oxide nanosheets using an eco-friendly mushroom extract strategy. Int. J. Nanomed. 2015, 10, 1505-1519.

22. Diekjürgen, D.; Grainger, D.W.; Diekjrgen, D. Polysaccharide matrices used in 3D in vitro cell culture systems. Biomaterials 2017, 141, 96-115. [CrossRef] [PubMed]

23. Rinaudo, M. Chitin and chitosan: Properties and applications. Prog. Polym. Sci. 2006, 31, 603-632. [CrossRef]

24. Younes, I.; Rinaudo, M. Chitin and Chitosan Preparation from Marine Sources. Structure, Properties and Applications. Mar. Drugs 2015, 13, 1133-1174. [CrossRef] [PubMed]

25. Domalik-Pyzik, P.; Chłopek, J.; Pielichowska, K. Chitosan-Based Hydrogels: Preparation, Properties, and Applications. In Polymers and Polymeric Composites: A Reference Series; Springer Science and Business Media LLC: Berlin/Heidelberg, Germany, 2019; pp. 1665-1693.

26. Croisier, F.; Jérôme, C. Chitosan-based biomaterials for tissue engineering. Eur. Polym. J. 2013, 49, 780-792. [CrossRef] 
27. Prasadh, S.; Suresh, S.; Wong, R. Osteogenic Potential of Graphene in Bone Tissue Engineering Scaffolds. Materials 2018, 11, 1430. [CrossRef] [PubMed]

28. Bohner, M.; Lemaître, J. Can bioactivity be tested in vitro with SBF solution? Biomaterials 2009, 30, $2175-2179$. [CrossRef] [PubMed]

29. Mezzetta, A.; Guazzelli, L.; Chiappe, C. Access to cross-linked chitosans by exploiting CO 2 and the double solvent-catalytic effect of ionic liquids. Green Chem. 2017, 19, 1235-1239. [CrossRef]

30. Posadowska, U.; Brzychczy-Wloch, M.; Pamula, E. Injectable gellan gum-based nanoparticles-loaded system for the local delivery of vancomycin in osteomyelitis treatment. J. Mater. Sci. Mater. Med. 2016, $27,9$. [CrossRef] [PubMed]

31. Xu, C.; Shi, X.; Ji, A.; Zhou, C.; Cui, Y. Fabrication and Characteristics of Reduced Graphene Oxide Produced with Different Green Reductants. PLoS ONE 2015, 10. [CrossRef]

32. Wang, Y.; Shi, Z.; Yin, J. Facile Synthesis of Soluble Graphene via a Green Reduction of Graphene Oxide in Tea Solution and Its Biocomposites. ACS Appl. Mater. Interfaces 2011, 3, 1127-1133. [CrossRef] [PubMed]

33. Liu, H.; Wu, J.; Liu, C.; Pan, B.; Kim, N.H.; Lee, J.H. Differently-charged graphene-based multilayer films by a layer-by-layer approach for oxygen gas barrier application. Compos. Part B: Eng. 2018, 155, 391-396. [CrossRef]

34. Depan, D.; Girase, B.; Shah, J.; Misra, R. Structure-process-property relationship of the polar graphene oxide-mediated cellular response and stimulated growth of osteoblasts on hybrid chitosan network structure nanocomposite scaffolds. Acta Biomater. 2011, 7, 3432-3445. [CrossRef] [PubMed]

35. Justin, R.; Chen, B. Characterisation and drug release performance of biodegradable chitosan-graphene oxide nanocomposites. Carbohydr. Polym. 2014, 103, 70-80. [CrossRef] [PubMed]

36. Gheisari, H.; Karamian, E.; Abdellahi, M. A novel hydroxyapatite-Hardystonite nanocomposite ceramic. Ceram. Int. 2015, 41, 5967-5975. [CrossRef]

37. Rubentheren, V.; Ward, T.A.; Chee, C.Y.; Tang, C.K. Processing and analysis of chitosan nanocomposites reinforced with chitin whiskers and tannic acid as a crosslinker. Carbohydr. Polym. 2015, 115, 379-387. [CrossRef]

38. Cervera, M.F.; Heinämäki, J.; de la Paz, N.; López, O.; Maunu, S.L.; Virtanen, T.; Hatanpää, T.; Antikainen, O.; Nogueira, A.; Fundora, J.; et al. Effects of Spray Drying on Physicochemical Properties of Chitosan Acid Salts. AAPS PharmSciTech 2011, 12, 637-649. [CrossRef]

39. Ogawa, K.; Yui, T.; Okuyama, K. Three D structures of chitosan. Int. J. Biol. Macromol. 2004, 34, 1-8. [CrossRef] 